\title{
Classifying 3 Moss Species by Deep Learning, Using the "Chopped Picture" Method
}

\author{
Takeshi Ise ${ }^{1,2 *}$, Mari Minagawa ${ }^{3}$, Masanori Onishi ${ }^{4}$ \\ ${ }^{1}$ Field Science Education and Research Center, Kyoto University, Kyoto, Japan \\ ${ }^{2}$ PRESTO, Japan Science and Technology Agency, Kawaguchi, Japan \\ ${ }^{3}$ Faculty of Agriculture, Kyoto University, Kyoto, Japan \\ ${ }^{4}$ Graduate School of Agriculture, Kyoto University, Kyoto, Japan \\ Email: *ise@kais.kyoto-u.ac.jp
}

How to cite this paper: Ise, T., Minagawa, M. and Onishi, M. (2018) Classifying 3 Moss Species by Deep Learning, Using the "Chopped Picture" Method. Open Journal of Ecology, 8, 166-173.

https://doi.org/10.4236/oje.2018.83011

Received: February 16, 2018

Accepted: March 17, 2018

Published: March 20, 2018

Copyright (C) 2018 by authors and Scientific Research Publishing Inc. This work is licensed under the Creative Commons Attribution-NonCommercial International License (CC BY-NC 4.0).

http://creativecommons.org/licenses/by-nc/4.0/

\begin{abstract}
Especially in recent years, deep learning has become a very effective tool for object identification. However, in general, the automatic object identification tends not to work well on ambiguous, amorphous objects such as vegetation. In this study, we developed a simple but effective approach to identify ambiguous objects and applied the method to several moss species. The technique called chopped picture method, where teacher images are systematically dissected into numerous small squares. As a result, the model correctly classified 3 moss species and "non-moss" objects in test images with accuracy more than $90 \%$. Using this approach will help progress in computer vision studies for various ambiguous objects.
\end{abstract}

\section{Keywords}

Remote Sensing, Classification, Deep Learning, Object Identification

\section{Introduction}

Especially in recent years, deep learning has become a very effective tool for object identification [1] [2]. However, deep learning is mostly applied for distinctive and deterministic objects such as human face, human body, cat, dog, automobile, etc. On the other hand, ambiguous objects such as trees, shrubs, and herbs are not very suitable for identification with machine learning.

In this study, we applied a new method called "chopped picture" to identify ambiguous, amorphous objects. In our case study, mosses (bryophytes), a type of green plants, are the target objects for identification. In general, differing from animals, plants have characteristics of modular growth. For example, numbers of 
leaves and stems which one individual typically possesses are not predetermined but change flexibly according to environmental conditions. This is clearly different that the standard model of human face has predetermined numbers of parts, such as two eyes, one mouth, and so on. These characteristics of plants can make object identification difficult.

Moreover, differing from flowering plants, mosses do not have vascular organs and their tissues are not well differentiated, and thus mosses are even more difficult target for machine learning. In addition, mosses tend to form mats consisted by numerous individuals of the same species. This behavior is challenging for object identification because it is very difficult to distinguish individuals. We willfully selected this difficult target for this case study, in order to test whether the "chopped picture" method (CPM) can overcome the difficulties. CPM is closely related to the patch-based object identification used for medical purposes [3].

\section{Method}

Kyoto, Japan is famous for beautiful moss gardens because its wet and moderately warm climate is suitable for moss growth. The study site is Murin-an, a traditional Japanese garden owned by Kyoto municipal government $(35.011557 \mathrm{~N}$, 135.787389E; Figure 1). The area of Murin-an is $3100 \mathrm{~m}^{2}$, and the climatic region is warm temperate forests. There are more than $50 \mathrm{spp}$. of mosses found in the previous study [4]. In this study, we selected 3 moss genus (Polytrichum species (POL), Trachycystis species (TRA), and Hypnum species (HYP)) for automatic identification using deep learning because these are the major moss genus in this garden. In addition to these 3 categories, we have another visual category "not moss (NOM)".

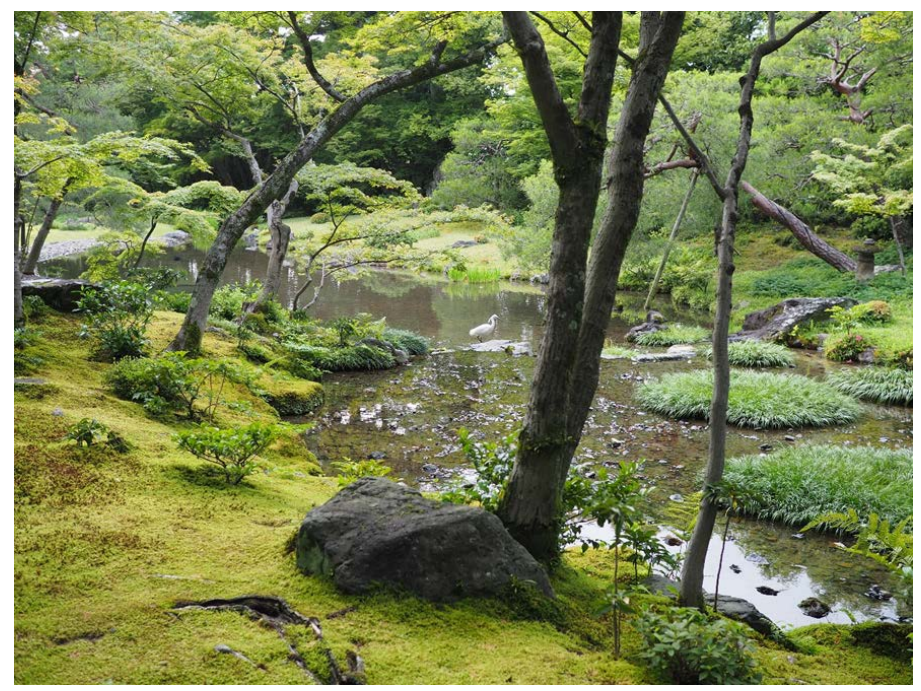

Figure 1. The scenery of Murin-an garden. The ground of this Japanese garden, the moisture level is relatively high due to rainfall and nearby ponds, and sunlight is moderately available due to sparsely planted trees. These environmental conditions make this garden a good habitat for several moss species. 
The training data is prepared by CPM in the following manner. First, in the garden, we located monotonous moss patches, which are uniformly covered by a single moss species, and took their pictures with digital camera (Figure 2). For example, the picture showing in Figure 2 has a pixel size of $4608 \times 3456$. Next, using R 3.3.2 [5], we "chopped" this picture into small squares $(56 \times 56)$ with $50 \%$ overlap both vertically and horizontally (Figure 3 ). With this method, it is very easy to obtain numerous teacher images because we simply need only a few digital photographs to make thousands of teacher images. We repeated this protocol for the 3 moss types (POL, TRA, and HYP) and "not moss" category (NOM). A small fraction of chopped pictures have "contaminations" with other types, and these impurities are screened and removed "by hand" easily. For the acquisition of image samples, we used Olympus OM-D E-M5 Mark II (16 mega pixels) and Panasonic LUMIX G 20 mm/F1.7 II ASPH (focal length of this lens is equivalent to $40 \mathrm{~mm}$ in $35 \mathrm{~mm}$ film), and took photographs directly above the moss patches by $60 \mathrm{~cm}$.

To make a model for object identification, we used the deep learning framework of nVIDIA DIGITS 4.0 [6] running on Ubuntu 14.04. The summary of the training is shown in Supplementary Figure S1. In total, we obtained 93,851 images for training and used $25 \%$ for validation. For the network model we used and the parameter settings, see Supplementary Table S1. The computer used for the training of computer vision was a BTO machine with Xeon E5-2603v3 (1.60 GHz, 6 cores), 16 GB RAM, and nVIDIA Quadro K620.

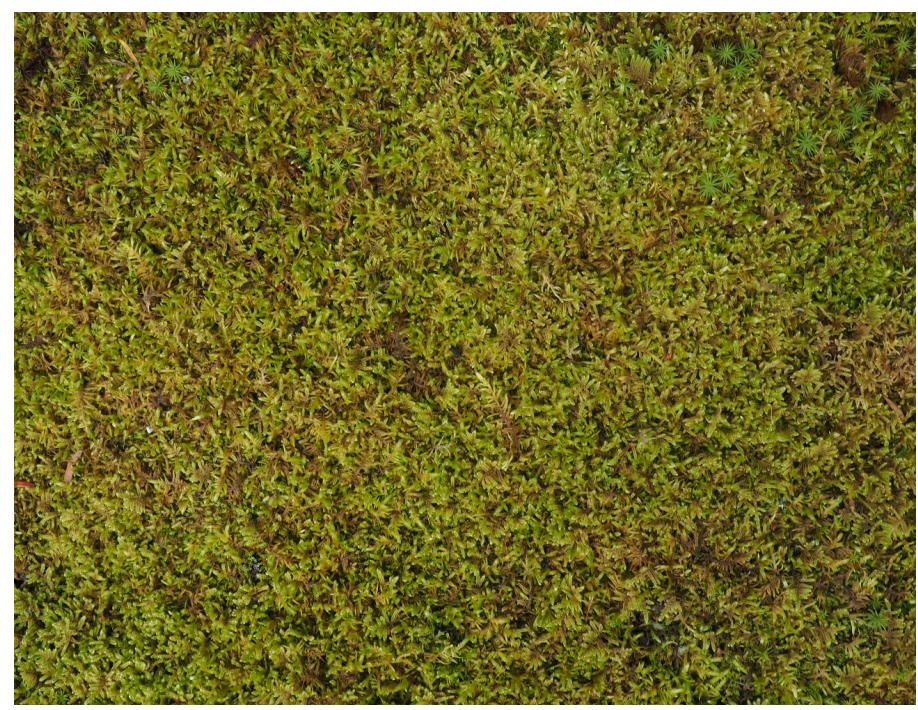

Figure 2. A photograph of the ground of Murin-an garden. Almost all area in this photograph is covered by Hypnum species (HYP). By chopping this large digital image $(4608 \times 3456$ pixels $)$ into small squares $(56 \times 56$ pixels) with $50 \%$ overlap, we were able to obtain a large training dataset for deep learning easily. This mat of Hypnum species has a small fraction of contamination with Polytrichum species. The small squares with the impurities are screened and removed "by hand". 

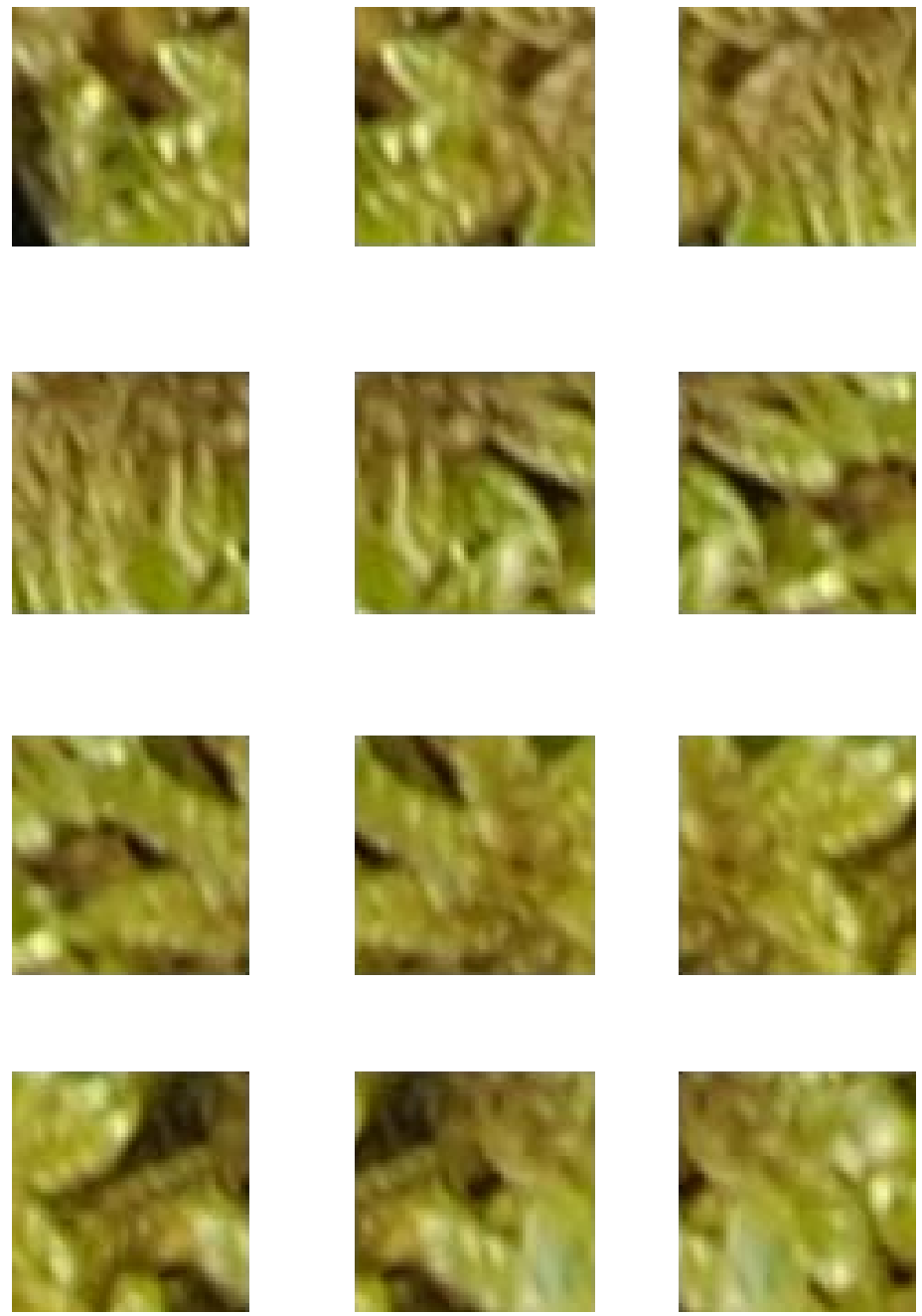

Figure 3. A set of examples of small squares $(56 \times 56$ pixels $)$ of the photograph of Hypnum species (HYP).

\section{Results and Discussion}

To test the performance of the trained model for object identification, we selected a picture which is also taken with the same protocol (focal length of 50 $\mathrm{mm}$ and $60 \mathrm{~cm}$ above the surface). In this picture, the target moss species and non-moss objects are included (Figure 4). To test the model, we also "chopped" this picture in the same manner as the training data. Using the model testing function of DIGITS 4.0, the model gives prediction and confidence of that prediction. Then we re-organize the small pieces into the original picture and showing predicted categories with colored circles (Figure 4). In this result, the model classified moss species appropriately. For detail, see the high-resolution image (Supplementary Figure S2). In the bottom right, the picture is mostly covered uniformly by POL, and the model nicely classified the objects. There are some growths of HYP within the POL patch, and the model has appropriately found HYP individuals.

The classification of TRA (mainly in the middle) and HYP (mainly in the left) 


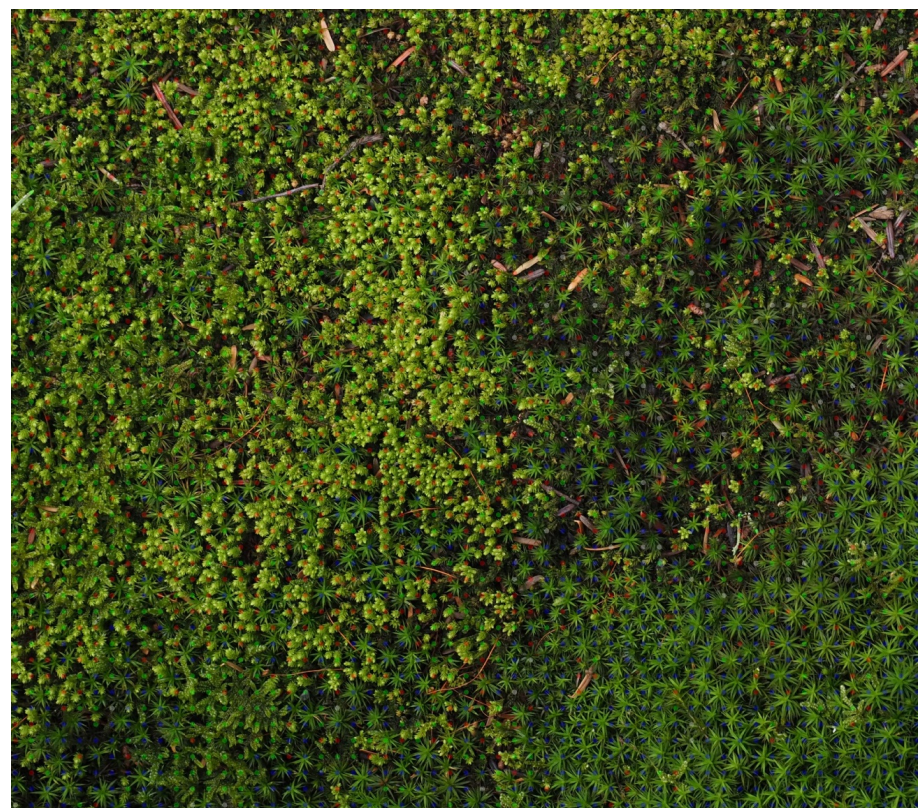

Figure 4. An example of model performance test with 3 moss species and "not moss" categories. The results of classification are color-coded (blue: POL, red: TRA, green: HYP, and white: NOM). If these color codes are difficult to see, please check the original image can be obtained in Supplementary Figure S2.

are generally appropriate, but with some classification errors. To obtain quantitative performance, we arbitrarily set regions where one moss species dominate. In those regions, from top left corner, we locate circles with the color of the dominated species, then evaluate whether the model prediction is correct or incorrect. We repeated this for 100 times for each region, and calculated the performance. The estimated performance for POL is $99 \%$, TRA is $95 \%$, and HYP is $74 \%$. Thus, CPM can be a good way to identify ambiguous objects such as green plants, especially mosses. Although mosses are highly amorphous, our method generally performed well.

However, the model performance was not the same for the 3 moss species. Identification of POL is excellent because this moss species is relatively large and has relatively distinctive, well-defined shape. On the other hand, the performance for HYP was not very good because this moss species is highly amorphous; it strongly shows vegetative growth with runners. As the result, the shape of HYP patches becomes amorphous and these characteristics affected the model performance.

To improve the model, we have the following suggestions. Color standardization can improve the performance. Color standardization using a color chart for photography will allow us to standardize the white balance and exposure. A trial and error for the "chopping size" should also be performed for the better performance. Although mosses are evergreen plants, they still show some seasonal differences in color, shape, and size. For example, spring is a growing season and TRA produces light green parts, easily visible for human eyes, in this season. 
Moreover, mosses produce reproductive organs in particular timings. Thus, collecting training data in many seasons and situations can improve the model.

Our results suggest several implications. Using CPM, we show that the performance of object identification with deep learning can be applied to things with amorphous shapes. This method can be applied for visual observations of plants in various scales, such as field photography (in this study), drone photography, aircraft photography such as Google Earth, and satellite images. In addition, as a practical application, we hope to make an application of "moss identifier" that allows people to know the moss names using a smartphone.

\section{Acknowledgements}

This work was a part of the Utilizing the Knowledge of Universities: a Multilateral Municipal Government Research Project, Kyoto City, Japan, and also supported by the JST PRESTO Grant Number JPMJPR15O1, Japan. We deeply appreciate professional advice from Drs. Yoshitaka Oishi and Wakana Azuma.

\section{References}

[1] Krizhevsky, A., Sutskever, I. and Hinton, G.E. (2012) ImageNet Classification with Deep Convolutional Neural Networks. Communications of the ACM, 60, 84-90. https://doi.org/10.1145/3065386

[2] Szegedy, C., Liu, W., Jia, Y., Sermanet, P., Reed, S., Anguelov, D., Erhan, D., Vanhoucke, V. and Rabinovich, A. (2015) Going Deeper with Convolutions. https://arxiv.org/pdf/1409.4842v1.pdf

[3] Wang, D., Khosla, A., Gargeya, R., Irshad, H. and Beck, A. (2016) Deep Learning for Identifying Metastatic Breast Cancer. https://arxiv.org/pdf/1606.05718v1.pdf

[4] Ueya Kato Landscape Co., Ltd. (2007) Annual Fostering Techniques of Existing Plants of Murin-An Garden as a National Place Scenic Beauty: For Preserving the Spatial Characteristics of Original Sensitivity of Aritomo Yamagata. Kyoto Municipal Government, Kyoto.

[5] R Core Team (2016) R: A Language and Environment for Statistical Computing. R Foundation for Statistical Computing. https://www.R-project.org/

[6] Barker, J. and Prasanna, S. (2016) Deep Learning for Object Detection with DIGITS. https://devblogs.nvidia.com/parallelforall/deep-learning-object-detection-digits/ 


\section{Supplementary Information}

\section{Job Directory}

/var/lib/digits/jobs/20170714-130600-f807

Disk Size

$361 \mathrm{MB}$

Netword (train/val)

train_val.prototxt

Network (deploy)

deploy.prototxt

Network (original)

original.prototxt

Solver

solver.prototxt

Raw caffe output

caffe_output.log

\section{Dataset}

murin.3

Done Fri Jul 14, 01:07:05 PM

Image Size
$56 \times 56$
Image Type
COLOR
DB backend
Imdb
Create DB (train)
70388 images
Create DB (val)
23463 images

Figure S1. Summary of training for object identification of 4 categories of moss garden using nVIDIA DIGITS 4.0.
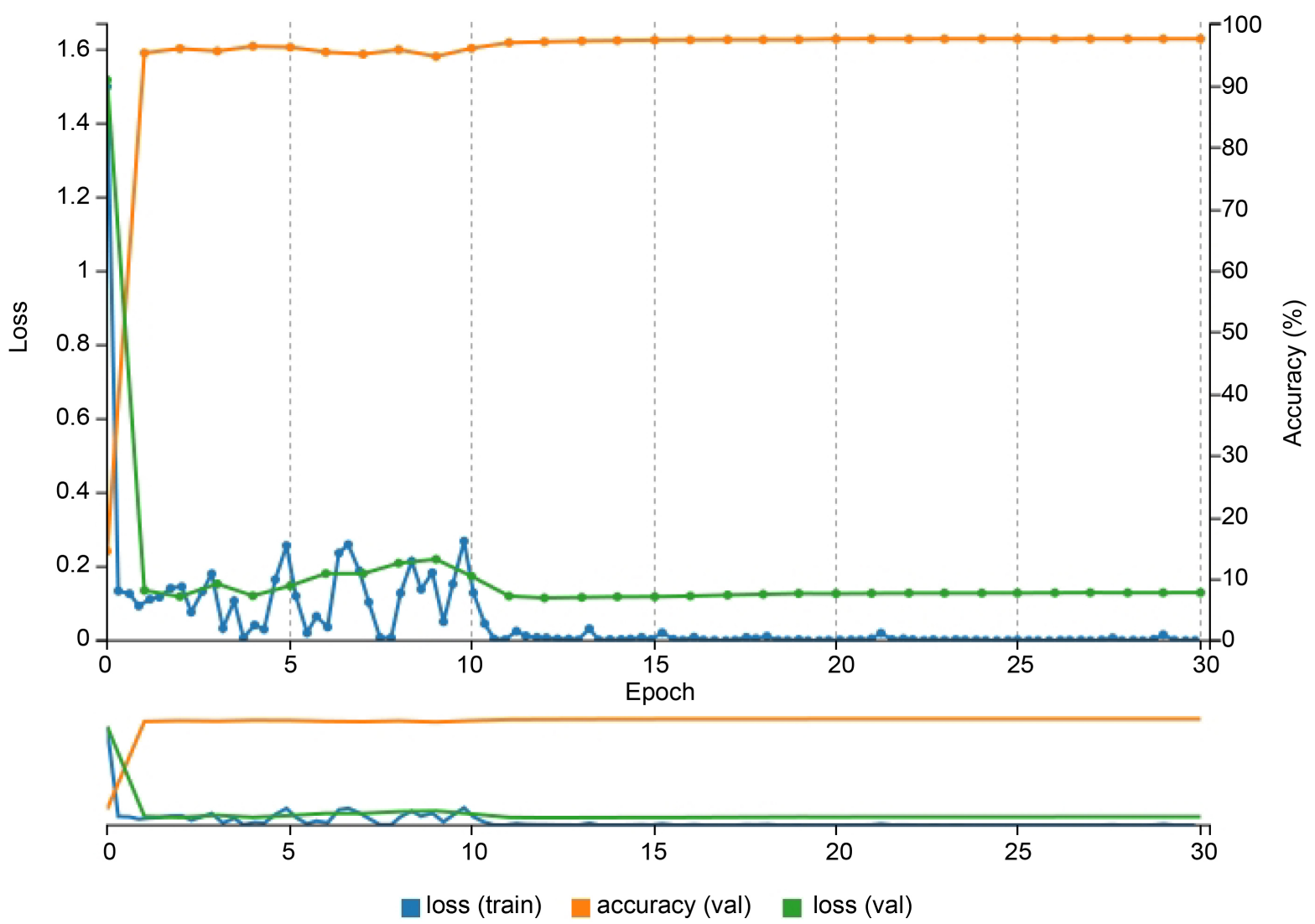

Figure S2. An example of model performance test with 3 moss species and "not moss" categories. The results of classification are color-coded (blue: POL, red: TRA, green: HYP, and white: NOM). 
Table S1. The setting of the nVIDIA DIGITS 4.0 image classification model.

\begin{tabular}{cc}
\hline Settings & Selected options \\
\hline Training epochs & 30 \\
Snapshot interval & 1 \\
Validation interval & 1 \\
Random seed & None \\
Batch size & Network defaults \\
Solver type & SGD \\
Base learning rate & 0.01 \\
Policy & Step down \\
Step size & 33 \\
Gamma & 0.1 \\
Network & LeNet \\
\hline
\end{tabular}

